\title{
Required concentration index quantifies effective drug combinations against hepatitis $C$ virus infection
}

\author{
Yusuke Kakizoe ${ }^{1,2+}$, Yoshiki Koizumi ${ }^{3 \dagger}$, Yukino Ikoma ${ }^{1}$, Hirofumi Ohashi ${ }^{4,5}$, Takaji Wakita ${ }^{4}$, Shingo Iwami ${ }^{1,6,7,8^{*}+}$ and \\ Koichi Watashi ${ }^{4,5,6,7,9 *+}$
}

\begin{abstract}
Successful clinical drug development requires rational design of combination treatments based on preclinical data. Anti-hepatitis $\mathrm{C}$ virus (HCV) drugs exhibit significant diversity in antiviral effect. Dose-response assessments can be used to determine parameters profiling the diverse antiviral effect during combination treatment. In the current study, a combined experimental and mathematical approaches were used to compare and score different combinations of anti-HCV treatments. A "required concentration index" was generated and used to rank the antiviral profile of possible double- and triple-drug combinations against HCV genotype $1 \mathrm{~b}$ and $2 \mathrm{a}$. Rankings varied based on target HCV genotype. Interestingly, multidrug (double and triple) treatment not only augmented antiviral activity, but also reduced genotype-specific efficacy, suggesting another advantage of multidrug treatment. The current study provides a quantitative method for profiling drug combinations against viral genotypes, to better inform clinical drug development.
\end{abstract}

Keywords: Mathematical model, Dose-response curve, Drug combination, Hepatitis C virus (HCV)

\section{Introduction}

Newly approved antiviral drugs rely upon dosage, treatment period, and drug combinations established during clinical trials. Trials require large cohorts of patients, significant cost, extensive time and strict management of ethics and compliance: Different dose regimens, treatment times and drug combinations are evaluated during trials $[1,2]$. Additional trials are needed to establish drug efficacy against different viral genotypes [3-6]. Despite the significant effort placed in clinical trials, escalation of dosage, increased treatment period, and combination

\footnotetext{
* Correspondence: siwami@kyushu-u.org; kwatashi@nih.go.jp

†Yusuke Kakizoe, Yoshiki Koizumi, Shingo Iwami and Koichi Watashi contributed equally to this work.

${ }^{1}$ Department of Biology, Faculty of Sciences, Kyushu University, Fukuoka 812-8581, Japan

${ }^{4}$ Department of Virology II, National Institute of Infectious Diseases, Tokyo 162-8640, Japan

Full list of author information is available at the end of the article
}

therapy, significant improvement in efficacy have not always been realized.

Drug concentrations which achieve 50\% virus reduction $\left(I C_{50}\right)$, can be used to characterize drug activity. Lower $I C_{50}$ means that antiviral effects are achieved with lower concentrations of drug [7]; however, a lower $I C_{50}$ does not necessarily translate to higher antiviral effect. Antiviral effect depends on the Hill coefficient $(m)$, in addition to $I C_{50}$. A higher $m$ value exponentially increases antiviral activity at higher doses [8-14]. We have previously shown that $m$ is unique to each anti-hepatitis $\mathrm{C}$ virus (HCV) drug, and that augmentation of antiviral activity with escalation of drug dose is quite diverse among the types of anti-HCV drugs [14]. Multidrug treatments also result in diverse effects depending on the drug combination. In-depth profiling of drug antiviral effects can be useful in designing a treatment protocol with maximal antiviral efficacy. Such profiling 
could result in significant savings in clinical trials. To date, antiviral efficacy variances between different anti$\mathrm{HCV}$ drugs and drug combinations has not been characterized in detail.

$\mathrm{HCV}$ infection is a leading cause of liver cirrhosis and hepatocellular carcinoma, serious public health problems affecting approximately 170 million people worldwide [15]. Recently, the development of new antiviral drugs known as direct acting antivirals (DAAs), have greatly improved treatment outcomes $[16,17]$. Commercial interests restrict the combinations which have entered clinical trials as the combinations are all company specific rather than based on any assessment of what would be the best combination for all available agents. Further evaluation of HCV DAA effects could help identify the "best" available therapy and assist with optimizing combination treatments. A new quantitative method could also support evaluation of next generation anti-HCV treatments that could lead to the eradication of HCV. In the current study, we compare antiviral profiles of different classes of anti$\mathrm{HCV}$ drugs to understand diversity of effects.

We recently developed a cell culture system combined with a mathematical model for quantifying anti-HCV drug efficacy at any concentration and multidrug combination [14]. We systematically evaluated and compared the intrinsic anti-HCV activity of 15 antiviral agents and their combinations against $\mathrm{HCV}$ genotype $1 \mathrm{~b}$. In the current study, we evaluate intrinsic anti-HCV activity in both genotype $1 \mathrm{~b}$ and $2 \mathrm{a}$. We create an "effectiveness" ranking for HCV replication inhibition in mono- and multi-drug cultures following exposure to high drug dose ranges. Significant diversity was observed between the antiviral activity profiles of different drugs. Thus, it is necessary to carefully select multidrug combinations to increase drug efficacy. We have demonstrated that the developed ranking index is able to delineate the advantages of past firstin-line anti-HCV treatment choices [14]. Thus, in the current study, we use the combined cell culture plus mathematical modeling approach to quantify efficacy of diverse antiviral drug combinations. This framework could be applied to other diseases requiring multidrug treatment, such as tuberculosis and cancer.

\section{Methods}

Anti-HCV effect of each drug against genotype $1 b$ or genotype 2a was evaluated with subgenomic replicon systems. As a genotype 1b model, LucNeo\#2 (LN2) cells were employed that carry a dicistronic subgenomic replicon including open reading frames (ORFs) for the firefly luciferase-neomycin phosphotransferase fusion protein (translated by $\mathrm{HCV} 5^{\prime}$-untranslated region) and the NS3-NS5B region of $\mathrm{HCV}$ genotype $1 \mathrm{~b}$ strain NN (translated by encephalomyocarditis virus (EMCV) internal ribosome entry site) [18]. Huh-7.5.1 cells transfected with a subgenomic replicon that included the ORFs for the NS3-NS5B region of HCV genotype 2a strain JFH-1 and the firefly luciferase gene (SGRJFH1/Luc) were used for a genotype 2a model [19]. These cells were seeded at $7 \times 10^{3}$ cells per well and treated with indicated concentrations of various drugs. Following $72 \mathrm{~h}$ of incubation, cells were lysed and cellular luciferase activity was measured to evaluate the HCV replication activity with a Luciferase Assay System (Promega) per manufacturer's protocol [18].

Fourteen anti-HCV drugs were evaluated as single treatments. Eleven of these were direct-acting antivirals (DAAs) of the following classes: NS3/4A protease inhibitors [PIs: telaprevir (TPV), danoprevir (DPV), simeprevir (SMV), and asunaprevir (ASV)], nucleoside NS5B polymerase inhibitor [NI: sofosbuvir (SOF)], non-nucleoside NS5B polymerase inhibitors [NNIs: VX-222 (VX), dasabuvir (DAS), nesbuvir (NSV), and tegobuvir (TGV)], and NS5A inhibitors [NS5AI: daclatasvir (DCV) and ledipasvir (LDV)]. The other 3 drugs tested were host-targeting agents (HTAs) including interferon-alpha (IFNa) and cyclophilin inhibitors [Cis: cyclosporin A (CsA) and SCY-635. For multidrug studies, cells were treated with combinations of two or three drugs prior to evaluation of activity. All anti-HCV agents were purchased or kindly provided as described [14].

\section{Results}

Fig. 1a provides a schematic of the combined experimental and mathematical system that we previously developed for quantifying anti-HCV activity of drug(s) [14]. In the previous study 14 anti-HCV agents were evaluated in mono and combination treatments against HCV genotype 1b [14]. In the current study the same 14 drugs (Table 1) were tested against HCV genotype $1 \mathrm{~b}$ (Fig. 1b) and HCV genotype 2a (Fig. 1c). Antiviral activity results from mono and combination treatments were used to develop a novel ranking index, the "required concentration index" or RCI (see below). Note that 14 anti-HCV agents include 11 direct-acting antivirals (DAAs) including NS3 protease inhibitors [PIs; telaprevir (TPV), danoprevir (DPV), simeprevir (SMV), and asunaprevir (ASV)], a nucleoside NS5B polymerase inhibitor [NI; sofosbuvir (SOF)], non-nucleoside NS5B polymerase inhibitors [NNIs; VX-222 (VX), dasabuvir (DAS), nesbuvir (NSV), and tegobuvir (TGV)], and NS5A inhibitors [NS5AI; daclatasvir (DCV) and ledipasvir (LDV)] and 3 host-targeting agents (HTAs) included interferon-alpha (IFNa) and cyclophilin inhibitors [CIs; cyclosporin A (CsA) and SCY-635 (SCY)].

\section{Ranking anti-HCV mono-drug treatments}

As shown in Fig. 1b, c, the antiviral profile of drugs against $\mathrm{HCV}$ genotypes $1 \mathrm{~b}$ and $2 \mathrm{a}$ vary widely, 


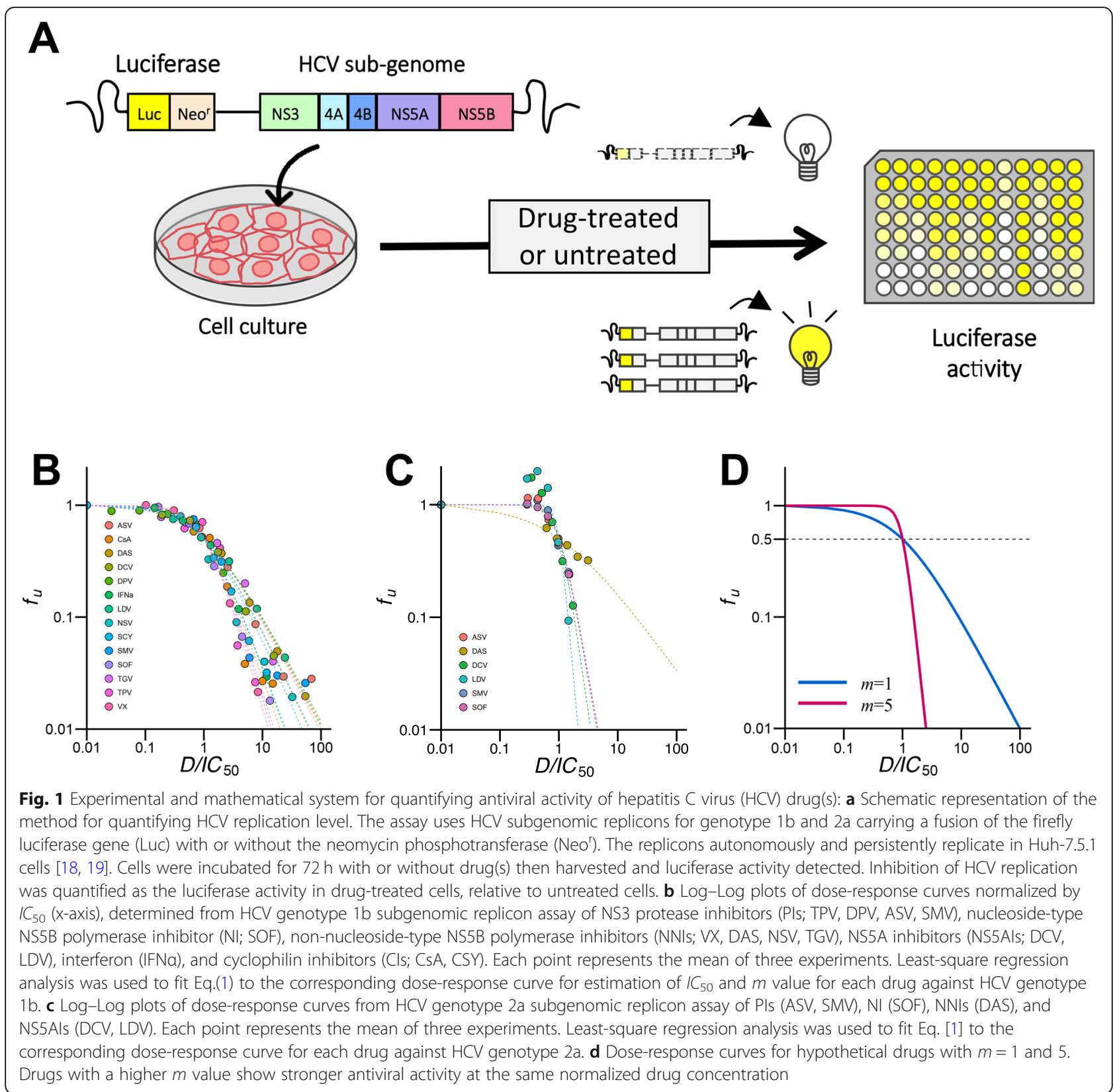

suggesting that anti-HCV drugs exhibit strain-dependent effects. The typical dose-response curves of a single antiviral drug can be analyzed using the following hill function [14] (Fig. 1d):

$$
f_{u}=\frac{1}{1+\left(\frac{D}{I C_{50}}\right)^{m}} .
$$

Here, $f_{u}$ represents the fraction of infection events unaffected by the drug (i.e., $1-f_{u}$ equals the fraction of drug-affected events). $D$ is the drug concentration, $I C_{50}$ is the drug concentration that achieves $50 \%$ inhibition of activity, and $m$ is the slope of the dose-response curve (i.e., Hill coefficient) [14]. Dose-response curves for drugs with higher $m$ values show stronger antiviral activity at the same normalized drug concentration so long as the drug concentration is higher than $I C_{50}$ (Fig. 1d). Least-square regression analysis was used to fit Eq.(1) to dose-response curves (Fig. $1 \mathrm{~b}, \mathrm{c}$ ) and estimate $I C_{50}$ and $m$ values. Estimated values for each drug against each HCV genotype are summarized in Table 1. The hill function may not accurately fit the dose-response curve at lower drug concentrations (Fig. 1c, especially for doses lower than $\left.I C_{50}\right)$. Typical clinical drug 
Table 1 Estimated characteristic parameters of the tested antiviral drugs

\begin{tabular}{|c|c|c|c|c|c|c|c|c|c|}
\hline \multirow[t]{2}{*}{ Drug } & \multirow[t]{2}{*}{ Type } & \multirow[t]{2}{*}{ Target } & \multirow[t]{2}{*}{ Class } & \multicolumn{2}{|l|}{$I C_{50}$} & \multicolumn{2}{|l|}{$m$} & \multicolumn{2}{|l|}{$\mathrm{RCl}_{95}$} \\
\hline & & & & genotype $1 b$ & genotype $2 a$ & genotype $1 b$ & genotype $2 a$ & genotype $1 b$ & genotype $2 a$ \\
\hline TPV (nM) & DAA & NS3 protease & PI & 323.79 & - & 1.72 & - & 5.54 & - \\
\hline DPV (nM) & DAA & NS3 protease & $\mathrm{PI}$ & 1.40 & - & 0.98 & - & 20.18 & - \\
\hline SMV (nM) & DAA & NS3 protease & $\mathrm{PI}$ & 0.45 & 153.95 & 1.10 & 3.10 & 14.54 & 2.59 \\
\hline ASV (nM) & DAA & NS3 protease & $\mathrm{PI}$ & 2.75 & 665.49 & 0.97 & 2.99 & 20.81 & 2.68 \\
\hline SOF (nM) & DAA & NS5B polymerase NI & $\mathrm{NI}$ & 120.48 & 843.74 & 1.66 & 3.02 & 5.89 & 2.65 \\
\hline VX (pM) & DAA & NS5B polymerase NNI & $\mathrm{NNI}$ & 107.58 & - & 1.81 & - & 5.08 & - \\
\hline DAS (nM) & DAA & NS5B polymerase NNI & $\mathrm{NNI}$ & 1.50 & 7203.98 & 0.99 & 0.73 & 19.57 & 56.39 \\
\hline NSV (nM) & DAA & NS5B polymerase NNI & $\mathrm{NNI}$ & 0.25 & - & 1.19 & - & 11.87 & - \\
\hline TGV (nM) & DAA & NS5B polymerase NNI & $\mathrm{NNI}$ & 8.92 & - & 1.01 & - & 18.45 & - \\
\hline $\mathrm{DCV}(\mathrm{nM})$ & DAA & NS5A & NS5Al & 0.10 & 0.13 & 1.11 & 3.68 & 14.19 & 2.23 \\
\hline LDV (nM) & DAA & NS5A & NS5Al & 0.67 & 30.80 & 0.96 & 6.11 & 21.48 & 1.62 \\
\hline IFNa (IU/ml) & HTA & - & IFN & 2.56 & - & 1.43 & - & 7.84 & - \\
\hline $\mathrm{Cs} A(\mu \mathrm{g} / \mathrm{m})$ & HTA & Cyclophilin & $\mathrm{Cl}$ & 0.40 & - & 1.53 & - & 6.85 & - \\
\hline $\mathrm{SCY}(\mu \mathrm{M})$ & HTA & Cyclophilin & $\mathrm{Cl}$ & 0.34 & - & 1.45 & - & 7.62 & - \\
\hline
\end{tabular}

concentrations are around 10- to 100 -fold of $I C_{50}$, therefore it is generally possible to quantify effectiveness of anti-HCV drug(s) with this method especially for such a high drug concentration. As discussed in recent publications [8-14], both $I C_{50}$ and $m$ values are needed to accurately estimate antiviral drug potency, though only $I C_{50}$ is widely used in the drug development field. Since estimated values for each drug differ relative to target HCV genotype, it is important to optimize mono and combination therapy against each genotype.

To characterize efficacy of drugs, we calculated a "required concentration index" (RCI) for each anti-HCV drug against genotype $1 \mathrm{~b}$ and $2 \mathrm{a}$. Assuming $1-f_{u}=x$ inhibition of viral replication, the $\mathrm{RCI}_{x}$ represents the critical fold increase of $I C_{50}$ requiring $x$ inhibition of viral replication. Solving Eq.(1) for $D / I C_{50}$, then $\mathrm{RCI}_{x}$ is represented as follows:

$$
\mathrm{RCI}_{x}=\frac{D_{x}}{I C_{50}}=\left(\frac{1}{f_{u}}-1\right)^{\frac{1}{m}}=\left(\frac{x}{1-x}\right)^{\frac{1}{m}}
$$

Here, $D_{x}$ is the drug concentration required to suppress $x$ of viral replication. Drugs with small $\mathrm{RCI}_{\mathrm{x}}$ values are more efficient inhibitors of $\mathrm{HCV}$ replication than drugs with high $\mathrm{RCI}_{x}$. Interestingly, high $m$ tends to be associated with smaller $\mathrm{RCI}_{\mathrm{x}}$. By substituting estimated $I C_{50}$ and $m$ parameters and setting $x$ to 0.95 in Eq.(2), we calculated the $\mathrm{RCI}_{\mathrm{x}}$ required for $95 \%$ inhibition of $\mathrm{HCV}$ replication (i.e., $\mathrm{RCI}_{95}$ ). We summarize $\mathrm{RCI}_{95}$ values of each drug against genotypes $1 b$ and $2 a$ in Fig. 2a, b, respectively. It should be noted that SOF, a nucleoside-type polymerase inhibitor used as a key agent in current and past DAA combinations, was effective in both genotype $1 \mathrm{~b}$ and $2 \mathrm{a}$, which is
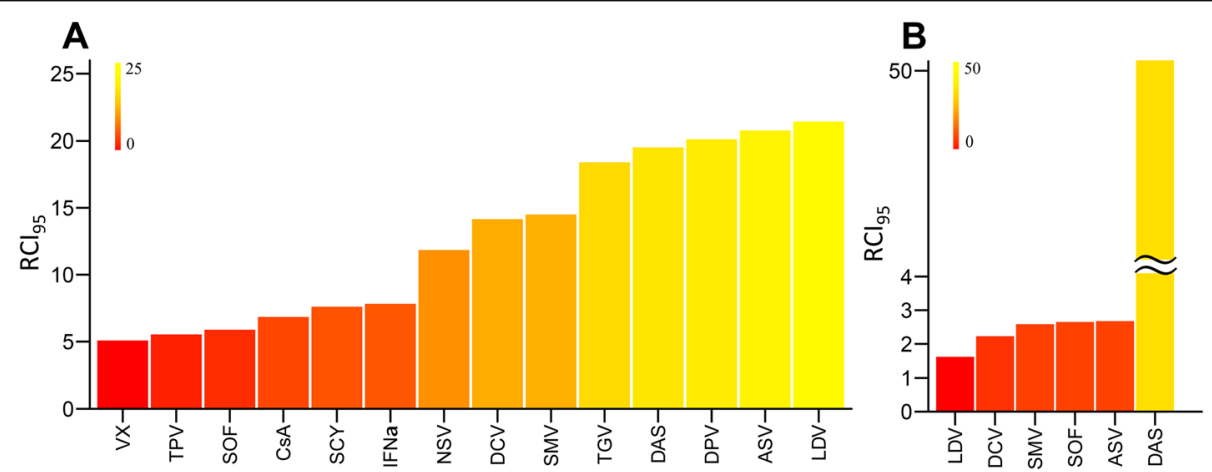

Fig. 2 Ranking anti-HCV mono drug treatments against genotypes $1 \mathrm{~b}$ and $2 \mathrm{a}$ : The critical dose of antiviral drug (i.e., fold increase of $/ C_{50}$ ) required to inhibit viral replication by $95 \%$, i.e., $\mathrm{RCl}_{95}$, was calculated for $\mathrm{HCV}$ (a) genotype $1 \mathrm{~b}$ and (b) genotype $2 \mathrm{a}$ 
consistent with SOF's known clinical pan-genotypic anti-HCV characteristic [20].

\section{Ranking anti-HCV multi-drug treatments}

Using the replicon system, the antiviral activity of doubleand triple-drug combinations (Fig. 3 \& Fig. 4) were investigated using consistent ratios of drug concentrations (i.e., $0.25 \times I C_{50}, 0.5 \times I C_{50}, 1 \times I C_{50}, 2 \times I C_{50}$, and $\left.4 \times I C_{50}\right)$.

Inhibitory activity was evaluated for 43 double drug combinations against $\mathrm{HCV}$ genotype $1 \mathrm{~b}$, and, 9 double drug combinations against genotype $2 \mathrm{a}$. Results are shown in Fig. 3a, b, respectively. Here, $D_{a}, D_{b}, \ldots, D_{i}$ are defined as the concentration of drug a, b, .., i and $I C_{50}^{a}$, $I C_{50}^{b}, \ldots, I C_{50}^{i}$ refer to the corresponding $I C_{50}$. Combined drug concentration in these experiments is described as $D^{\text {com }}$ $=\left(D_{a}, D_{b}, \ldots, D_{i}\right)=\left(\tilde{D} \times I C_{50}^{a}, \tilde{D} \times I C_{50}^{b}, \ldots, \tilde{D} \times I C_{50}^{i}\right)$, where $\tilde{D}=D_{a} / I C_{50}^{a}=D_{b} / I C_{50}^{b}=\ldots=D_{i} / I C_{50}^{i}$ is the constant ratio to $I C_{50}$ of each combined drug (x-axis of dose-response curves). As shown in Fig. 3c, a similar hill function can be fit to dose-response curves of drug combinations [14]:

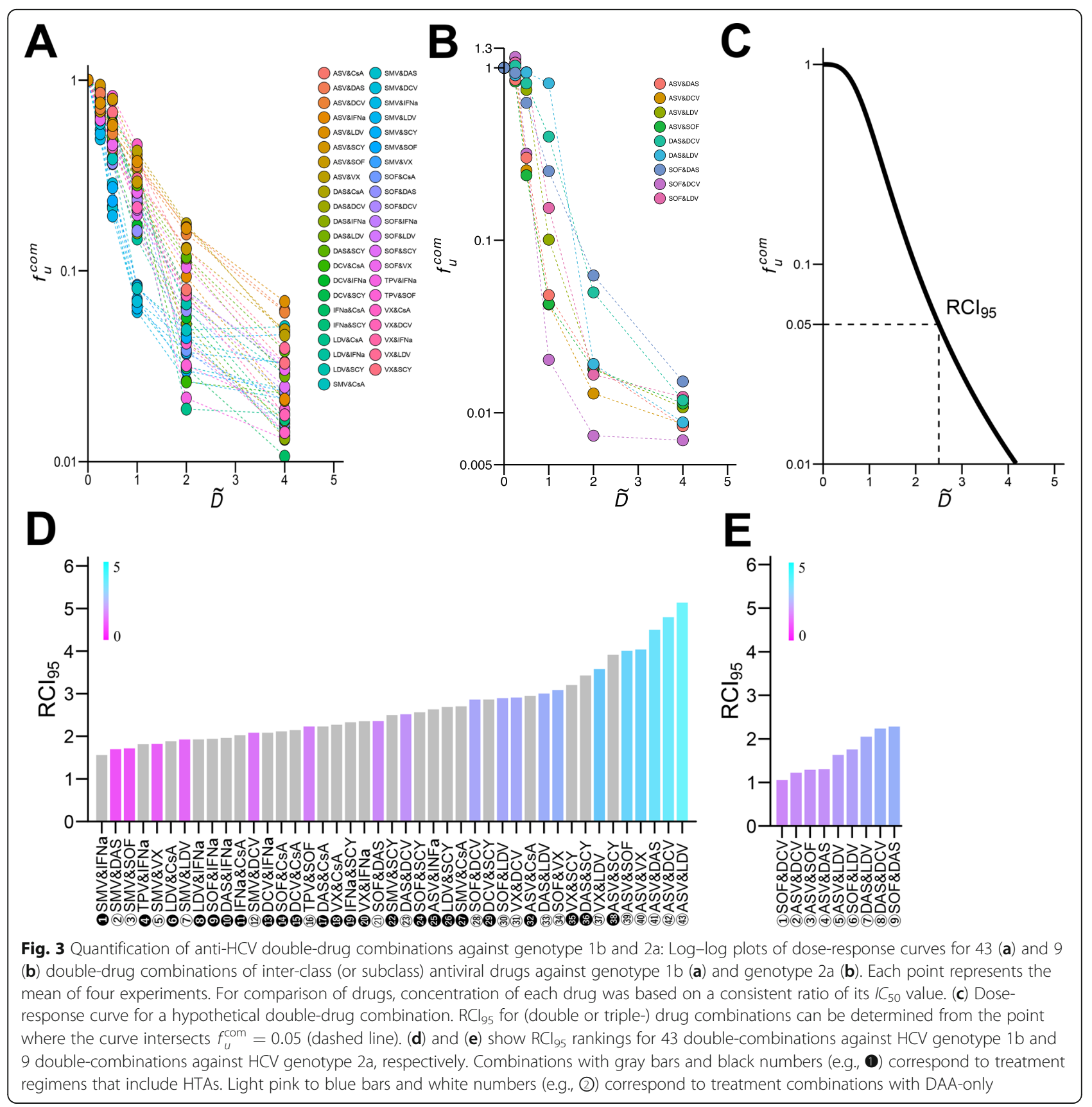


A
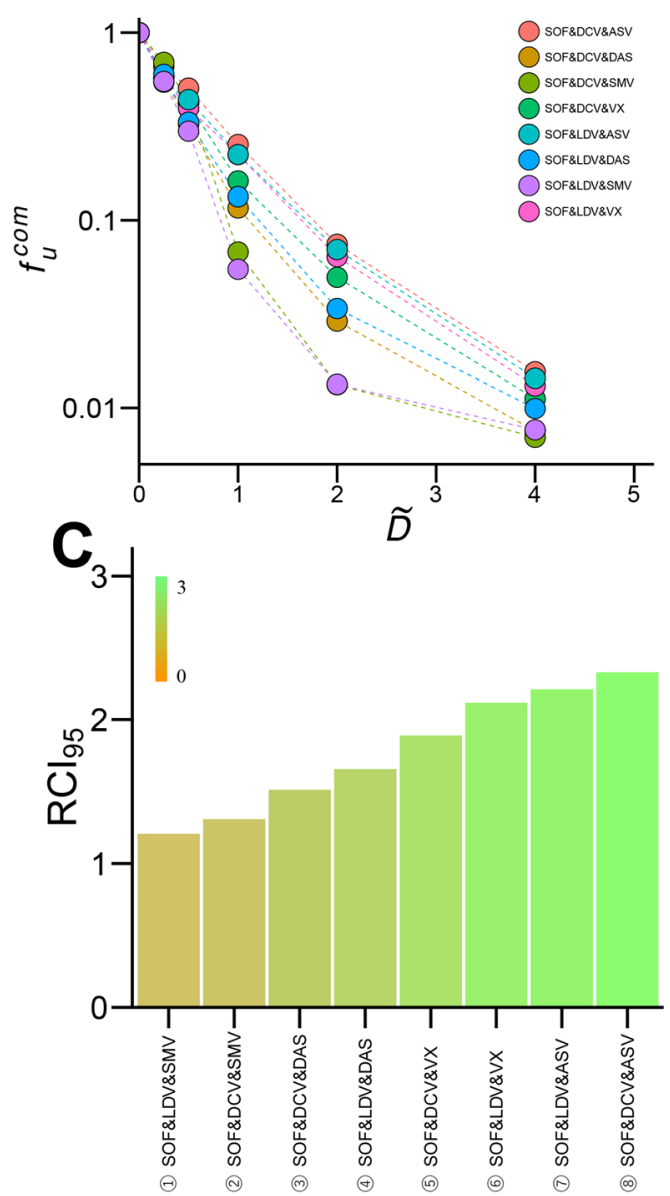

B
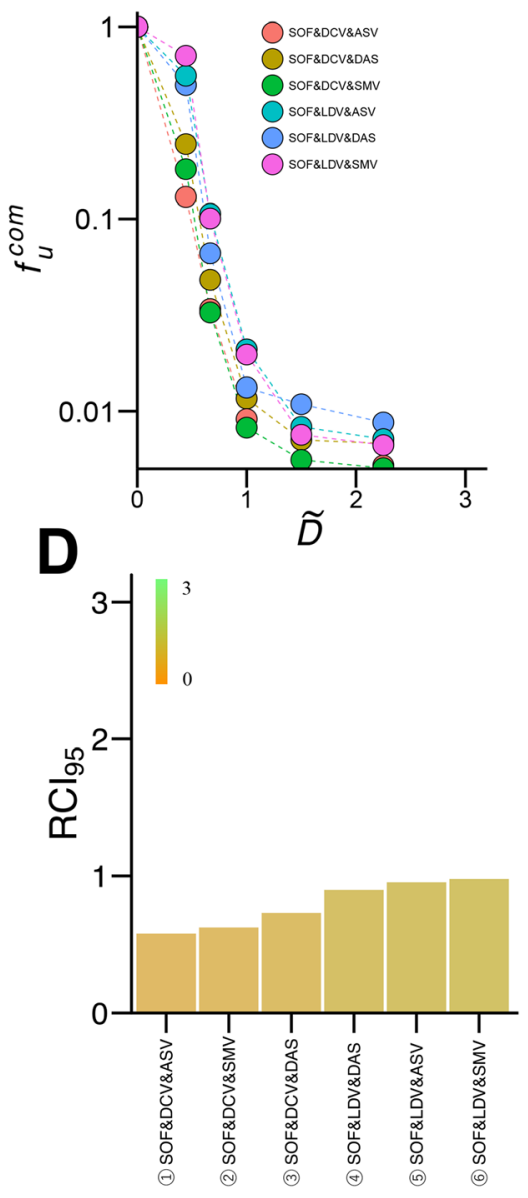

Fig. 4 Quantification of anti-HCV triple-drug combinations against genotype 1b and 2a: Log-log plots of dose-response curves for 8 (a) and 6 (b) triple-drug combinations against HCV genotype $1 b(\mathbf{a})$ and $2 a(\mathbf{b})$. Each point represents the mean of five experiments for genotype $1 \mathrm{~b}$ and four experiments for genotype 2a. For comparison of drugs, concentration of each drug was based on a consistent ratio of its $/ \mathcal{C}_{50}$ value. (c) and $(\mathbf{d})$ show $\mathrm{RCl}_{95}$ rankings of the triple-drug combinations against HCV genotype $1 \mathrm{~b}$ and $2 \mathrm{a}$, respectively

$$
f_{u}^{\mathrm{com}}=\frac{1}{1+\left(\frac{\tilde{D}}{I C_{50}^{\text {com }}}\right)^{m^{\mathrm{com}}} .}
$$

Here, $f_{u}^{\text {com }}$ is the fraction of infection events unaffected by the drug combination, $I C_{50}^{\text {com }}$ is the constant ratio that inhibits $\mathrm{HCV}$ replication by $50 \%$, and $m^{\text {com }}$ is the Hill coefficient [14]. In Table 2, we summarize estimated parameters, $I C_{50}^{\text {com }}$ and $m^{\text {com }}$, for double-drug combinations.

Similar to mono treatments, the required concentration index for drug combinations is derived as

$$
\begin{aligned}
\mathrm{RCI}_{x} & =\tilde{D}_{c}=I C_{50}^{\mathrm{com}}\left(\frac{1}{f_{c}^{\mathrm{com}}}-1\right)^{\frac{1}{m^{\mathrm{com}}}} \\
& =I C_{50}^{\mathrm{com}}\left(\frac{x}{1-x}\right)^{\frac{1}{m^{\mathrm{com}}}}
\end{aligned}
$$

The $\mathrm{RCI}_{95}$ required for $95 \%$ inhibition of HCV replication is extrapolated from the point at which the curve intersects $f_{u}^{\text {com }}=0.05$ (dashed line in Fig. 3c). Note that the critical constant ratio, $\tilde{D}_{c}$, satisfying Eq.(4) can be uniquely determined. The $\mathrm{RCI}_{95}$ values for double-drug combinations against genotype $1 \mathrm{~b}$ and $2 \mathrm{a}$ are summarized in Fig. $3 \mathrm{~d}$, e, respectively. $\mathrm{RCI}_{95}$ varies depending on drug combination. For genotype $1 \mathrm{~b}, \mathrm{RCI}_{95}$ ranged from 1.56 to 5.14 , for genotype $2 \mathrm{a} \mathrm{RCI}_{95}$ ranged from 1.05 to 2.28. The drug combination with the best anti$\mathrm{HCV}$ profile against genotype $1 \mathrm{~b}$ is SMV plus IFNa; Fig. $3 \mathrm{~d}$ (1) This combination used to be the first-in-line anti-HCV drug prior to the development of DAA treatments [17]. Combinations including a non-DAA are presented as gray bars with black number designations. Combinations with DAA-only double treatments are plotted in light pink to blue and designated with white 
Table 2 Estimated characteristic parameters of the antiviral drug combinations

\begin{tabular}{|c|c|c|c|c|c|c|}
\hline \multirow[t]{2}{*}{ Drug } & \multicolumn{2}{|l|}{$I C_{50}^{\text {com }}$} & \multicolumn{2}{|l|}{$m^{\mathrm{com}}$} & \multicolumn{2}{|l|}{$\mathrm{RCl}_{95}$} \\
\hline & genotype $1 b$ & genotype $2 a$ & genotype $1 b$ & genotype $2 a$ & genotype $1 b$ & genotype $2 a$ \\
\hline ASV\&CsA & 0.80 & - & 2.25 & - & 2.95 & - \\
\hline ASV\&DAS & 0.61 & 0.28 & 1.48 & 1.94 & 4.50 & 1.30 \\
\hline ASV\&DCV & 0.59 & 0.27 & 1.41 & 1.97 & 4.79 & 1.22 \\
\hline ASV\&IFNa & 0.53 & - & 1.84 & - & 2.63 & - \\
\hline ASV\&LDV & 0.65 & 0.47 & 1.43 & 2.30 & 5.14 & 1.63 \\
\hline ASV\&SCY & 0.88 & - & 1.97 & - & 3.91 & - \\
\hline ASV\&SOF & 0.54 & 0.24 & 1.47 & 1.75 & 4.01 & 1.29 \\
\hline ASV\&VX & 0.77 & - & 1.78 & - & 4.04 & - \\
\hline DAS\&CsA & 0.60 & - & 2.23 & - & 2.23 & - \\
\hline DAS\&DCV & 0.47 & 0.78 & 1.75 & 2.80 & 2.52 & 2.24 \\
\hline DAS\&IFNa & 0.40 & - & 1.84 & - & 1.96 & - \\
\hline DAS\&LDV & 0.54 & 0.86 & 1.72 & 3.41 & 3.00 & 2.05 \\
\hline DAS\&SCY & 0.57 & - & 1.64 & - & 3.43 & - \\
\hline DCV\&CsA & 0.50 & - & 2.02 & - & 2.14 & - \\
\hline DCV\&IFNa & 0.40 & - & 1.79 & - & 2.08 & - \\
\hline DCV\&SCY & 0.53 & - & 1.75 & - & 2.86 & - \\
\hline IFNa\&CsA & 0.67 & - & 2.67 & - & 2.02 & - \\
\hline IFNa\&SCY & 0.59 & - & 2.14 & - & 2.33 & - \\
\hline LDV\&CsA & 0.43 & - & 1.99 & - & 1.88 & - \\
\hline LDV\&IFNa & 0.38 & - & 1.82 & - & 1.93 & - \\
\hline LDV\&SCY & 0.52 & - & 1.79 & - & 2.69 & - \\
\hline SMV\&CsA & 0.23 & - & 1.20 & - & 2.70 & - \\
\hline SMV\&DAS & 0.18 & - & 1.31 & - & 1.70 & - \\
\hline SMV\&DCV & 0.20 & - & 1.26 & - & 2.08 & - \\
\hline SMV\&IFNa & 0.17 & - & 1.34 & - & 1.56 & - \\
\hline SMV\&LDV & 0.14 & - & 1.14 & - & 1.92 & - \\
\hline SMV\&SCY & 0.19 & - & 1.14 & - & 2.50 & - \\
\hline SMV\&SOF & 0.20 & - & 1.38 & - & 1.71 & - \\
\hline SMV\&VX & 0.24 & - & 1.45 & - & 1.82 & - \\
\hline SOF\&CsA & 0.55 & - & 2.18 & - & 2.12 & - \\
\hline SOF\&DAS & 0.34 & 0.62 & 1.53 & 2.25 & 2.36 & 2.28 \\
\hline SOF\&DCV & 0.47 & 0.27 & 1.64 & 2.14 & 2.86 & 1.05 \\
\hline SOF\&IFNa & 0.37 & - & 1.77 & - & 1.94 & - \\
\hline SOF\&LDV & 0.42 & 0.52 & 1.52 & 2.43 & 2.89 & 1.76 \\
\hline SOF\&SCY & 0.50 & - & 1.80 & - & 2.56 & - \\
\hline SOF\&VX & 0.47 & - & 1.57 & - & 3.09 & - \\
\hline TPV\&IFNa & 0.46 & - & 2.14 & - & 1.81 & - \\
\hline TPV\&SOF & 0.64 & - & 2.37 & - & 2.23 & - \\
\hline VX\&CsA & 0.77 & - & 2.71 & - & 2.27 & - \\
\hline VX\&DCV & 0.47 & - & 1.61 & - & 2.91 & - \\
\hline VX\&IFNa & 0.47 & - & 1.83 & - & 2.35 & - \\
\hline VX\&LDV & 0.59 & - & 1.63 & - & 3.58 & - \\
\hline VX\&SCY & 0.76 & - & 2.05 & - & 3.20 & - \\
\hline
\end{tabular}


numbers (Fig. 3d). For the DAA-only combinations, one of the most effective treatments against genotype $1 \mathrm{~b}$ was the combination of SMV and SOF (Fig. 3d(3), a primary treatment choice in the early era of DAA-only treatment [16]. A long term first-in-line DAA combination, SOF and LDV (Fig. 3d(30), e(6), ranked in the mid-range of efficacy against both genotype $1 \mathrm{~b}$ and 2a. Most other drug combinations ranked differently against genotype $1 \mathrm{~b}$ and genotype 2a. ASV plus LDV (Fig. 3d(43), e(5) was the least effective DAA-only combination against genotype $1 \mathrm{~b}$, but fell in the mid-range for effectiveness against genotype 2a. SOF plus DAS (Fig. 3d(21), e(9) ranked in the mid-range against genotype $1 \mathrm{~b}$, but ranked lowest against genotype $2 \mathrm{a}$. These trends suggest an overall difference in drug effect depending on the target $\mathrm{HCV}$ genotype, and indicate the importance of profiling drugs against each genotype.

Eight triple-DAA treatments were profiled against HCV genotype $1 \mathrm{~b}$ and 6 triple-combinations were evaluated against genotype 2a (Fig. 4a, b). Triple combination assessments included NS3 protease inhibitor (SMV, ASV) with NS5A inhibitor (DCV, LDV) and NI NS5B polymerase inhibitor (SOF), or NS5A inhibitor with NI NS5B polymerase inhibitor and NNI NS5B polymerase inhibitor (VX, DAS). $I C_{50}^{\text {com }}$ and $m^{\text {com }}$ for triple-drug combinations are summarized in Table 3. We need to note that our experimental assay can detect the range of $0.005<f_{u}^{\text {com }}<0.01$ in Fig. 4a, b, whereas it is difficult to measure $f_{u}^{\text {com }}<0.005$ in areas of higher drug concentration, reaching to the detection limit of the assay. $\mathrm{RCI}_{95}$ values of triple-drug combinations against genotype $1 \mathrm{~b}$ and $2 \mathrm{a}$ are summarized in Fig. 4c, d, respectively. $\mathrm{RCI}_{95}$ values ranged from 1.21 to 2.33 for genotype $1 \mathrm{~b}$ and 0.58 to 0.98 for genotype 2a. Triple combination treatment with SOF, LDV and SMV was most effective against genotype $1 \mathrm{~b}$ (Fig. 4c(1)), and least effective against genotype 2a (Fig. 4d(6). SOF plus DCV and SMV (Fig. 4c(2) was also significantly effective against genotype $1 \mathrm{~b}$, consistent with the reported clinical efficacy of this triple combination [21, 22]. These results show the optimal combination of drugs to suppress viral replication in vitro, and shed light on the promising drug combinations for improving clinical outcome.

The correlation in ranking between the required concentration index and clinical data suggest that this method could assist with the search for drugs that achieve an efficient antiviral inhibition with different $\mathrm{HCV}$ genotypes.

\section{Discussion}

Our study shows that the concentration of drug (calculated as fold of $I C_{50}$ ), that achieves $95 \%$ virus inhibition $\left(\mathrm{RCI}_{95}\right)$, highly varied depending on the type of drug and combination with other drugs. $\mathrm{RCI}_{95}$ of drugs in mono treatment ranged as much as 4.2 fold in antiviral activity against $\mathrm{HCV}$ genotype $1 \mathrm{~b}$ (Fig. $2 \mathrm{a}, \mathrm{RCI}_{95}=5.08-21.4$ ). This diversity in $\mathrm{RCI}_{95}$ indicates the importance of characterizing more than just the $\mathrm{IC}_{50}$ of drugs when predicting antiviral efficacy in clinical settings. In double-drug combinations, $\mathrm{RCI}_{95}$ values decreased (Fig. 3d, e) compared with mono treatments (Fig. 2a, b), indicating elevated antiviral activity resulted from combination treatment. The $\mathrm{RCI}_{95}$ values of DAA-only double combinations ranged from 1.70 (SMV \& DAS) to 5.14 (ASV \& LDV) in genotype $1 \mathrm{~b}$ and from 1.05 (SOF \& DCV) to 2.28 (SOF \& DAS) in genotype 2a. Thus, the diversity in $\mathrm{RCI}_{95}$ is different among genotypes. Genotype differences are probably due differences in replication activity and the varied dependency on target [23, 24].

Triple DAA treatments have become the final strategy for improving treatment outcomes, especially with difficult-to-treat HCV. Triple combinations are also used as a means to shorten treatment periods. Understanding the activity of triple DAA combinations is important in advancing towards worldwide eradication of $\mathrm{HCV}$ virus [25-28]. Consistent with ongoing clinical trials which show higher treatment efficacy of triple-drug combinations, triple combinations reduced $\mathrm{RCI}_{95}$ beyond double-

Table 3 Estimated characteristic parameters of the antiviral drug combinations

\begin{tabular}{|c|c|c|c|c|c|c|}
\hline \multirow[t]{2}{*}{ Drug } & \multicolumn{2}{|l|}{$I C_{50}^{\text {com }}$} & \multicolumn{2}{|l|}{$m^{\text {com }}$} & \multicolumn{2}{|l|}{$\mathrm{RCl}_{95}$} \\
\hline & genotype $1 \mathrm{~b}$ & genotype $2 a$ & genotype $1 b$ & $\overline{\text { genotype } 2 \mathrm{a}}$ & genotype $1 \mathrm{~b}$ & genotype $2 a$ \\
\hline SOF\&DCV\&VX & 0.40 & - & 1.92 & - & 1.89 & - \\
\hline SOF\&DCV\&ASV & 0.55 & 0.13 & 2.03 & 2.09 & 2.33 & 0.58 \\
\hline SOF\&DCV\&DAS & 0.34 & 0.20 & 1.97 & 2.28 & 1.52 & 0.73 \\
\hline SOF\&DCV\&SMV & 0.33 & 0.16 & 2.14 & 2.23 & 1.31 & 0.62 \\
\hline SOF\&LDV\&VX & 0.45 & - & 1.90 & - & 2.12 & - \\
\hline SOF\&LDV\&ASV & 0.46 & 0.33 & 1.88 & 2.88 & 2.21 & 0.95 \\
\hline SOF\&LDV\&DAS & 0.35 & 0.26 & 1.88 & 2.49 & 1.66 & 0.90 \\
\hline SOF\&LDV\&SMV & 0.26 & 0.35 & 1.90 & 3.04 & 1.21 & 0.98 \\
\hline
\end{tabular}


drug combination levels (Fig. 4c, d). $\mathrm{RCI}_{95}$ for triple drug combinations ranged from 1.21 to 2.33 in genotype $1 \mathrm{~b}$ and from 0.58 to 0.98 in genotype 2a. Interestingly, the $\mathrm{RCI}_{95}$ values of selected drugs (SMV, ASV, DCV, LDV, DAS, VX and SOF) were less variable in triple-drug combinations compared with double combinations. $\mathrm{RCI}_{95}$ ranged 1.9 fold (1.21 for SOF \& LDV \& SMV to 2.33 for SOF \& DCV \& ASV) with triple-drug combinations, 3.0 fold (1.70 for SMV \& DAS to 5.14 for ASV \& LDV) in double-drug combinations, and 4.2 fold (5.08 for VX to 21.4 for LDV) in single-drug treatment against genotype $1 \mathrm{~b}$. These data suggest that multidrug treatments such as triple-drug combinations provide more consistent antiviral effect irrespective of the choice of drugs, yet another advantage of triple combinations.

\section{Conclusion}

In an era of rapidly progressing anti-HCV treatments, selection of the "best" combination treatment is critical to establishing the next generation of anti-HCV treatments against difficult-to-treat $\mathrm{HCV}$ and eventually eradicating $\mathrm{HCV}$. We have developed an integrated experimental and mathematical method to evaluate the efficacy of anti-HCV dugs against $\mathrm{HCV}$ genotype $1 \mathrm{~b}$ and 2a. The method was used to score mono- and multidrug treatment regimens against $\mathrm{HCV}$. This scoring could be used to optimize multidrug treatment regimens prior to clinical entry.

\section{Abbreviations \\ HCV: Hepatitis C virus; DAA: Direct acting antiviral; RCI: Required concentration index; TPV: Telaprevir; DPV: Danoprevir; SMV: Simeprevir; ASV: Asunaprevir; SOF: Sofosbuvir; VX: VX-222; DAS: Dasabuvir; NSV: Nesbuvir; TGV: Tegobuvir; DCV: Daclatasvir; LDV: Ledipasvir; IFNa: Interferon-alpha; CsA: Cyclosporin A; SCY: SCY-635}

\section{Acknowledgements}

We are grateful to Dr. Kunitada Shimotohno at National Center for Global Health and Medicine for providing LucNeo\#2 cells. SCY635 was kindly provided by Scynexis, Inc. We appreciate the editorial assistance provided by Dr. Senko Tsukuda at Department of Virology II, National Institute of Infectious.

\section{Authors' contributions}

$\mathrm{SI}$ and $\mathrm{KW}$ designed the experiments. $\mathrm{HO}$ and $\mathrm{KW}$ conducted the experiments. YK, YK and YI carried out the computational analyses. SI and KW supervised the project. All authors contributed to the manuscript text. The author(s) read and approved the final manuscript.

\section{Funding}

This study was supported in part by Grants-in-Aid for JSPS Scientific Research (KAKENHI) Scientific Research B 18KT0018 (to S.I.), 18 H01139 (to S.I), 16H04845 (to S.I.), 17H04085 (to K.W.), Scientific Research in Innovative Areas $19 H 04839$ (to S.I.), 18 H05103 (to S.I.); AMED CREST $19 \mathrm{~g} 1310002$ (to S.I.); AMED J-PRIDE $19 f \mathrm{fm} 0208006 \mathrm{s0103}$ (to S.I.), 19fm0208014h0003 (to S.I.),

$19 f m 0208019 h 0103$ (to S.I.), 19fm0208019j0003 (to K.W.); AMED Research Program on HIV/AIDS $19 f k 0410023 s 0101$ (to S.I.); Research Program on Emerging and Re-emerging Infectious Diseases 19fk0108050h0003 (to S.I.); Program for Basic and Clinical Research on Hepatitis 19fk0210036h0502 (to S.I.), $19 f k 0210036 j 0002$ (to K.W.); Program on the Innovative Development and the Application of New Drugs for Hepatitis B 19fk0310114h0103 (to S.I.), 19fk0310114j0003 (to K.W.), 19fk0310101j1003 (to K.W.), 19fk0310103j0203 (to
K.W.); JST MIRAI (to S.I. and K.W.); JST CREST (to S.I. and K.W.); Mitsui Life Social Welfare Foundation (to S.I. and K.W.); Shin-Nihon of Advanced Medical Research (to S.I.); Suzuken Memorial Foundation (to S.I.); Life Science Foundation of Japan (to S.I.); SECOM Science and Technology Foundation (to S.I.); The Japan Prize Foundation (to S.I.); Toyota Physical and Chemical Research Institute (to S.I.); Fukuoka Financial Group, Inc. (to S.I.); Kyusyu Industrial Advancement Center Gapfund Program (to S.I.); Foundation for the Fusion Of Science and Technology (to S.I.); The Yasuda Medical Foundation (to K.W.); Smoking Research Foundation (to K.W.); Takeda Science Foundation (to K.W.); Mochida Memorial Foundation for Medical and Pharmaceutical Research (to K.W.).

\section{Availability of data and materials}

All data generated or analyzed during this study are included in this published article.

Ethics approval and consent to participate

Not applicable.

\section{Consent for publication}

Not applicable.

\section{Competing interests}

The authors declare that they have no competing interests.

\section{Author details}

'Department of Biology, Faculty of Sciences, Kyushu University, Fukuoka 812-8581, Japan. ${ }^{2}$ Present address: Data Science Group, Advanced Technology Division, INTAGE Inc, Tokyo 101-8201, Japan. ${ }^{3}$ National Center for Global Health and Medicine, Tokyo 162-8655, Japan. ${ }^{4}$ Department of Virology II, National Institute of Infectious Diseases, Tokyo 162-8640, Japan.

${ }^{5}$ Department of Applied Biological Science, Tokyo University of Science, Noda 278-8510, Japan. ${ }^{6}$ Institute for the Advanced Study of Human Biology (ASHBi), Kyoto University, Kyoto 606-8501, Japan. ${ }^{7}$ NEXT-Ganken Program, Japanese Foundation for Cancer Research (JFCR), Tokyo 135-8550, Japan. ${ }^{8}$ Science Groove Inc, Fukuoka, Japan. ${ }^{9}$ Institute for Frontier Life and Medical Sciences, Kyoto University, Kyoto, Japan.

Received: 9 June 2020 Accepted: 10 December 2020

Published online: 09 January 2021

\section{References}

1. Pawlotsky JM. New hepatitis C therapies: the toolbox, strategies, and challenges. Gastroenterology. 2014;146(5):1176-92.

2. Schinazi $R$, Halfon $P$, Marcellin $P$, Asselah T. HCV direct-acting antiviral agents: the best interferon-free combinations. Liver Int. 2014;34(Suppl 1):69-78.

3. Afdhal N, Zeuzem S, Kwo P, Chojkier M, Gitlin N, Puoti M, et al. Ledipasvir and sofosbuvir for untreated HCV genotype 1 infection. N Engl J Med. 2014; 370(20):1889-98.

4. Gane EJ, Hyland RH, Yang Y, Svarovskaia E, Stamm LM, Brainard DM, et al. Efficacy of Ledipasvir plus Sofosbuvir for 8 or 12 weeks in patients with hepatitis C virus genotype 2 infection. Gastroenterology. 2017:152(6):1366-71.

5. Gane EJ, Hyland RH, An D, Svarovskaia E, Pang PS, Brainard D, et al. Efficacy of ledipasvir and sofosbuvir, with or without ribavirin, for 12 weeks in patients with HCV genotype 3 or 6 infection. Gastroenterol. 2015;149(6): 1454-61.e1.

6. Kohli A, Kapoor R, Sims Z, Nelson A, Sidharthan S, Lam B, et al. Ledipasvir and sofosbuvir for hepatitis $C$ genotype 4: a proof-of-concept, single-Centre, open-label phase 2a cohort study. Lancet Infect Dis. 2015;15(9):1049-54.

7. Aykul S, Martinez-Hackert E. Determination of half-maximal inhibitory concentration using biosensor-based protein interaction analysis. Anal Biochem. 2016;508:97-103.

8. Shen L, Peterson S, Sedaghat AR, McMahon MA, Callender M, Zhang H, et al. Dose-response curve slope sets class-specific limits on inhibitory potential of anti-HIV drugs. Nat Med. 2008;14(7):762-6.

9. Sampah ME, Shen L, Jilek BL, Siliciano RF. Dose-response curve slope is a missing dimension in the analysis of HIV-1 drug resistance. Proc Natl Acad Sci U S A. 2011;108(18):7613-8. 
10. Shen L, Rabi SA, Siliciano RF. A novel method for determining the inhibitory potential of anti-HIV drugs. Trends Pharmacol Sci. 2009;30(12):610-6.

11. Jilek BL, Zarr M, Sampah ME, Rabi SA, Bullen CK, Lai J, et al. A quantitative basis for antiretroviral therapy for HIV-1 infection. Nat Med. 2012;18(3):446-51.

12. Shen L, Rabi SA, Sedaghat AR, Shan L, Lai J, Xing S, et al. A critical subset model provides a conceptual basis for the high antiviral activity of major HIV drugs. Sci Transl Med. 2011;3(91):91 ra63.

13. Laskey SB, Siliciano RF. A mechanistic theory to explain the efficacy of antiretroviral therapy. Nat Rev Microbiol. 2014;12(11):772-80.

14. Koizumi Y, Ohashi H, Nakajima S, Tanaka Y, Wakita T, Perelson AS, et al. Quantifying antiviral activity optimizes drug combinations against hepatitis C virus infection. Proc Natl Acad Sci U S A. 2017;114(8):1922-7.

15. Lauer GM, Walker BD. Hepatitis C virus infection. N Engl J Med. 2001; 345(1):41-52.

16. Pawlotsky JM. Hepatitis $C$ treatment: the data flood goes on-an update from the liver meeting 2014. Gastroenterology. 2015;148(3):468-79.

17. Pawlotsky JM, Feld JJ, Zeuzem S, Hoofnagle JH. From non-a, non-B hepatitis to hepatitis C virus cure. J Hepatol. 2015;62(1 Suppl):S87-99.

18. Goto K, Watashi K, Murata T, Hishiki T, Hijikata M, Shimotohno K. Evaluation of the anti-hepatitis $C$ virus effects of cyclophilin inhibitors, cyclosporin a, and NIM811. Biochem Biophys Res Commun. 2006:343(3):879-84.

19. Kato T, Date T, Miyamoto M, Sugiyama M, Tanaka Y, Orito E, et al. Detection of anti-hepatitis $C$ virus effects of interferon and ribavirin by a sensitive replicon system. J Clin Microbiol. 2005;43(11):5679-84.

20. Feld JJ, Jacobson IM, Hezode C, Asselah T, Ruane PJ, Gruener N, et al. Sofosbuvir and Velpatasvir for HCV genotype 1, 2, 4, 5, and 6 infection. N Engl J Med. 2015;373(27):2599-607.

21. Lau G, Benhamou Y, Chen G, Li J, Shao Q, Ji D, et al. Efficacy and safety of 3-week response-guided triple direct-acting antiviral therapy for chronic hepatitis C infection: a phase 2, open-label, proof-of-concept study. Lancet Gastroenterol Hepatol. 2016;1(2):97-104.

22. Sulkowski MS, Feld JJ, Lawitz E, Felizarta F, Corregidor AM, Khalid O, et al. Efficacy and safety of 6 or 8 weeks of simeprevir, daclatasvir, sofosbuvir for HCV genotype 1 infection. J Viral Hepat. 2018;25(6):631-9.

23. Scheel TK, Gottwein JM, Mikkelsen LS, Jensen TB, Bukh J. Recombinant HCV variants with NS5A from genotypes 1-7 have different sensitivities to an NS5A inhibitor but not interferon-alpha. Gastroenterology. 2011;140(3): 1032-42.

24. McPhee F, Sheaffer AK, Friborg J, Hernandez D, Falk P, Zhai G, et al. Preclinical profile and characterization of the hepatitis $C$ virus NS3 protease inhibitor Asunaprevir (BMS-650032). Antimicrob Agents Chemother. 2012; 56(10):5387-96.

25. Kohli A, Osinusi A, Sims Z, Nelson A, Meissner EG, Barrett LL, et al. Virological response after 6 week triple-drug regimens for hepatitis $C$ : a proof-ofconcept phase 2A cohort study. Lancet. 2015;385(9973):1107-13.

26. Everson GT, Sims KD, Rodriguez-Torres M, Hezode C, Lawitz E, Bourliere M, et al. Efficacy of an interferon- and ribavirin-free regimen of daclatasvir, asunaprevir, and BMS-791325 in treatment-naive patients with HCV genotype 1 infection. Gastroenterology. 2014;146(2):420-9.

27. Poordad F, Sievert W, Mollison L, Bennett M, Tse E, Brau N, et al. Fixed-dose combination therapy with daclatasvir, asunaprevir, and beclabuvir for noncirrhotic patients with HCV genotype 1 infection. Jama. 2015;313(17): $1728-35$.

28. Muir AJ, Poordad F, Lalezari J, Everson G, Dore GJ, Herring R, et al. Daclatasvir in combination with asunaprevir and beclabuvir for hepatitis $C$ virus genotype 1 infection with compensated cirrhosis. Jama. 2015;313(17): 1736-44.

\section{Publisher's Note}

Springer Nature remains neutral with regard to jurisdictional claims in published maps and institutional affiliations.

Ready to submit your research? Choose BMC and benefit from:

- fast, convenient online submission

- thorough peer review by experienced researchers in your field

- rapid publication on acceptance

- support for research data, including large and complex data types

- gold Open Access which fosters wider collaboration and increased citations

- maximum visibility for your research: over $100 \mathrm{M}$ website views per year

At $\mathrm{BMC}$, research is always in progress.

Learn more biomedcentral.com/submissions 